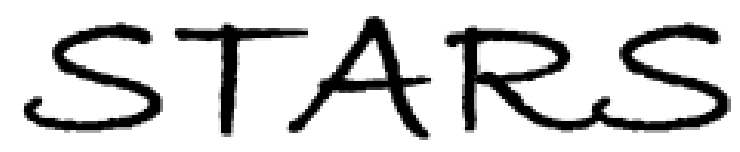

University of Central Florida

STARS

$1-1-2009$

\title{
Design considerations for surface plasmon resonance-based fiber-optic detection of human blood group
}

Anuj K. Sharma

Rajan Jha

Himansu S. Pattanaik

University of Central Florida

Gerhard J. Mohr

Find similar works at: https://stars.library.ucf.edu/facultybib2000

University of Central Florida Libraries http://library.ucf.edu

This Article is brought to you for free and open access by the Faculty Bibliography at STARS. It has been accepted for inclusion in Faculty Bibliography 2000s by an authorized administrator of STARS. For more information, please contact STARS@ucf.edu.

\section{Recommended Citation}

Sharma, Anuj K.; Jha, Rajan; Pattanaik, Himansu S.; and Mohr, Gerhard J., "Design considerations for surface plasmon resonance-based fiber-optic detection of human blood group" (2009). Faculty Bibliography 2000s. 2125.

https://stars.library.ucf.edu/facultybib2000/2125

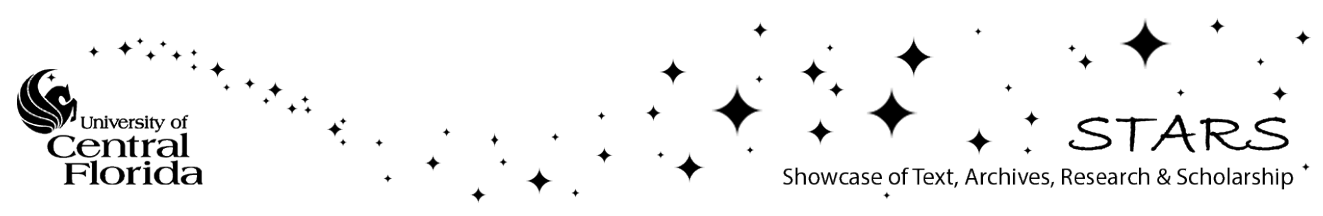




\section{Design considerations for surface plasmon resonance-based fiber-optic detection of human blood group}

\author{
Anuj K. Sharma*,+ \\ Friedrich-Schiller University \\ Institute of Physical Chemistry \\ 10 Lessingstrasse \\ Jena, 07743 \\ Germany \\ E-mail: anuj.sharma@uni-jena.de
}

\section{Rajan Jha*}

Carrer de la Coruna

13 Mediterranean Technology Park

Castelldefels, Barcelona 08860

Spain

Himansu S. Pattanaik

University of Central Florida

College of Optics and Photonics (CREOL)

Orlando, Florida 32816

\section{Gerhard J. Mohr}

Friedrich-Schiller University

Institute of Physical Chemistry

10 Lessingstrasse

Jena 07743 Germany

\begin{abstract}
A fiber-optic surface plasmon resonance (SPR) sensor for the detection of human blood groups is proposed. Previous experimental results describing the wavelength-dependent refractive index variation of multiple samples of different blood groups are considered for theoretical calculations. The spectral interrogation method, along with silica fiber and silver layer, is considered. The sensor's performance is closely analyzed in terms of shift in SPR wavelength and SPR curve width in order to optimize the design parameters for a reliable and accurate blood-group identifier. The sensor design parameters include silver layer thickness, fiber core diameter, sensing region length, and temperature variation. The results are explained in terms of light coupling and plasmon resonance condition. The proposed sensing probe is able to provide high sensitivity and accuracy of blood-group detection, thereby opening an easy and reliable window for medical applications. ( 2009 Society of Photo-Optical Instrumentation Engineers. [DOI: 10.1117/1.3275476]
\end{abstract}

Keywords: blood-group sensor; plasmon; optical fiber; sensitivity.

Paper 09164R received Apr. 26, 2009; revised manuscript received Oct. 5, 2009; accepted for publication Oct. 22, 2009; published online Dec. 28, 2009.

\section{Background}

Surface plasmon resonance (SPR) is one of the promising optical techniques that find several applications in different fields. The SPR phenomenon was first exploited in 1978 for characterization of thin films by Pockrand et al. ${ }^{1}$ However, the first sensing application of SPR was presented by Liedberg and Nylander in $1982 .^{2}$ In the last two decades, SPR-based optical fiber sensors have been utilized in sensing a wide range of physical and biochemical parameters. ${ }^{3-5}$ Under Kretchmann and Reather's attenuated total reflection (ATR) configuration, ${ }^{6}$ a p-polarized light of wavelength $\lambda$ satisfies the resonance condition and excites a charge density oscillation known as surface plasmon wave (SPW). The plasmon resonance condition is expressed as:

$$
K_{0} n_{c} \sin \theta=K_{0}\left(\frac{\varepsilon_{m r} n_{s}^{2}}{\varepsilon_{m r}+n_{s}^{2}}\right)^{1 / 2} ; \quad K_{0}=\frac{2 \pi}{\lambda} .
$$

The term on the left-hand side is the propagation constant $\left(K_{E W}\right)$ of the evanescent wave generated due to ATR of light incident at an angle $\theta$ through the light coupling device (e.g., optical fiber) of refractive index $n_{c}$. The right-hand term is the SPW propagation constant $\left(K_{S P W}\right)$, with $\varepsilon_{m r}$ being the real

\footnotetext{
*Authors contributed equally as first authors

${ }^{+}$Address all correspondence to: Anuj K. Sharma, Friedrich-Schiller University, Institute of Physical Chemistry, 10 Lessingstrasse, Jena, 07743 Germany. Tel: 49-364-194-8355; Fax: 49-364-194-8302; E-mail: anuj.sharma@uni-jena.de.
}

part of the metal dielectric constant $\left(\varepsilon_{m}\right)$ and $n_{s}$ the refractive index of the sensing (dielectric) medium. When the preceding condition is fulfilled, the resonance appears in the form of a sharp dip of output signal $(P)$ at a resonance wavelength $\left(\lambda_{S P R}\right)$ due to strong optical absorption by SPW (Fig. 1). Any change in $n_{s}$ near the metal-dielectric interface causes a shift in the value of $\lambda_{S P R}$. The performance of the SPR sensor is determined in terms of two aspects. First, the shift in resonance wavelength $\left(\delta \lambda_{S P R}\right)$ for a given change $\left(\delta n_{s}\right)$ in $n_{s}$ should be as large as possible. Second, the full width at half maximum (FWHM) corresponding to SPR curves should be as small as possible for precise measurements.

Among various biological parameters, SPR-based sensing has been reported for the detection of pesticides, ${ }^{5}$ membrane proteins, ${ }^{7}$ immunoassays, ${ }^{8}$ DNA, RNA, and allergens. However, the fiber-optic SPR sensor can also be a potential candidate for efficient detection of human blood groups. The logic behind this reasoning is that the different blood groups have different dispersion relationships due to their different chemical and biological compositions. Therefore, in view of Eq. (1), the plasmon resonance condition should be satisfied at different $\lambda_{S P R}$ values for different blood groups, thereby making it possible to realize a simple and reliable SPR determination of blood groups.

$1083-3668 / 2009 / 14(6) / 064041 / 6 / \$ 25.00$ ○ 2009 SPIE 


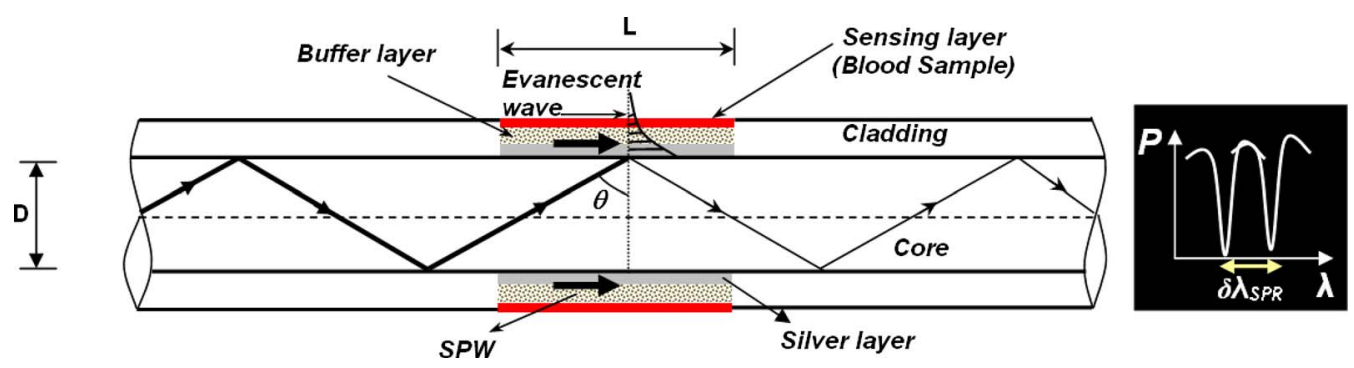

Fig. 1 Proposed fiber-optic SPR sensor probe setup for the detection of human blood groups.

Li et al. ${ }^{9}$ experimentally measured the refractive dispersion of three blood groups $(\mathrm{O}, \mathrm{A}$, and $\mathrm{B})$ at visible and nearinfrared (NIR) wavelengths $(380 \mathrm{~nm}$ to $860 \mathrm{~nm})$ for a number of blood samples. Based on their experimental results, they described the refractive dispersion for three blood groups in the form of a Cauchy formula given as

$$
n(\lambda)=1.357+\frac{A}{\lambda^{2}}+\frac{B}{\lambda^{4}} .
$$

In the preceding expression, wavelength $(\lambda)$ is in $\mathrm{nm}$. The Cauchy coefficients can have different values for different blood groups. Based on the fitting of data points of experimental dispersion curves reported by Li et al., ${ }^{9}$ Fig. 2 shows the variation of refractive index for three different blood groups $(\mathrm{O}, \mathrm{A}$, and $\mathrm{B})$ with wavelength. As is apparent, the trends of the curves (i.e., decrease in refractive index with an increase in wavelength) are in accordance with the normal dispersion shown by most of the SPR-active liquid media (e.g., water, etc.), which gives a first-hand indication that SPR sensing can be made possible for blood samples also. The data corresponding to the plot shown in Fig. 2 have been used for simulation in the present work.

The present state-of-the-art suggests that despite being heavily used for almost three decades, the SPR sensing principle is still unexplored for the detection of human blood groups. Therefore, in the present work, we have explored the possibility of designing a fiber-optic SPR blood-group sensor by making use of the experimental data provided by Li et al. ${ }^{9}$ We report the design considerations to enable a fiber-optic SPR sensor for detection of different blood groups. Standard fused silica fiber coated with thin SPR-active silver (Ag) layer has been considered for the proposed scheme. The spectral interrogation method of SPR sensing is used. The influence of critical design parameters such as metal layer thickness, ratio of sensing region length to fiber core diameter $(L / D)$, and temperature is studied on the proposed sensor's performance in order to identify the best possible working conditions leading to highly accurate and reliable SPR-based fiber-optic detection of different blood groups.

\section{Design Considerations}

In this section, we systematically discuss the modalities of different constituents of the proposed sensor design along with their physical, biological, and optical properties.

\subsection{Optical Fiber}

The coupling device is considered a multimode optical fiber (of core diameter $D$ ), whose wavelength-dependent refractive index $\left(n_{c}\right)$ is represented in terms of the Sellmeier expression, as follows ${ }^{10}$ :

$$
n_{c}(\lambda)=\left(1+\frac{A_{1} \lambda^{2}}{\lambda^{2}-B_{1}^{2}}+\frac{A_{2} \lambda^{2}}{\lambda^{2}-B_{2}^{2}}+\frac{A_{3} \lambda^{2}}{\lambda^{2}-B_{3}^{2}}\right)^{1 / 2},
$$

where $\lambda$ denotes the wavelength (in $\mu \mathrm{m}$ ). The coefficients $A_{1}$, $A_{2}, A_{3}, B_{1}, B_{2}$, and $B_{3}$ are known as Sellmeier coefficients and have certain numeric values for fused silica. ${ }^{10}$ The numerical aperture (NA) of a multimode fiber is generally in the vicinity of 0.20 .

\subsection{Metal Layer}

As shown in Fig. 1, a small portion (of length $L$ ) of fiber cladding is removed and is coated with a thin Ag layer of thickness $d$. According to the free-electron Drude model, the wavelength-dependent complex dielectric function $\left(\varepsilon_{m}\right)$ of $\mathrm{Ag}$ can be written as:

$$
\varepsilon_{m}(\lambda)=\varepsilon_{m r}+i \varepsilon_{m i}=1-\frac{\lambda^{2} \lambda_{c}}{\lambda_{p}^{2}\left(\lambda_{c}+i \lambda\right)} .
$$

In the preceding expression, $\lambda_{p}$ stands for plasma wavelength, and $\lambda_{c}$ stands for collision (or damping) wavelength. For Ag, the standard value of $\lambda_{p}$ is $145.41 \mathrm{~nm}$ and of $\lambda_{c}$ is $17.61 \mu \mathrm{m}$ (Ref. 11).

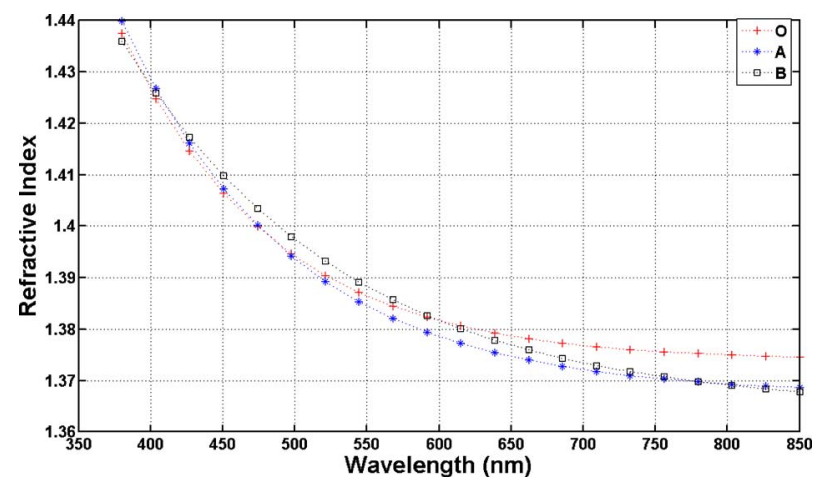

Fig. 2 Plot for dispersion (refractive index versus wavelength) of samples corresponding to three different blood groups. 


\subsection{Buffer Layer}

The Ag layer in Fig. 1 is followed by a buffer layer of thickness in the vicinity of 1 to $15 \mathrm{~nm}$ with refractive index of 1.45 (Ref. 11). Preferably, this buffer layer should be in the form of a biochemical layer for two important reasons. First, it prevents the blood sample from being in direct contact with the Ag layer, which may contaminate the blood sample, thereby affecting sensor's performance. The contamination due to the presence of the Ag layer may arise due to the fact that silver is prone to oxidation problems when used in liquid environments. If inappropriately exposed to liquids, Ag may form a thin ( 2 to $3 \mathrm{~nm}$ ) oxide layer on its surface. Such an oxide layer may lead to errors in SPR measurements, and for this reason, the Ag layer must be protected against such oxidation issues. Therefore, one may choose a biochemical layer that can act as a protective layer to keep the Ag layer from being oxidized. Second, the structural compatibility of the blood sample with such a biochemical layer is another added advantage. Structural compatibility lies in the fact that due to having both chemical and biological characteristics, a single biochemical layer may provide stable bonding at the two interfaces (one with the Ag layer and another with the blood sample) to prevent any structural anomaly at those interfaces (e.g., polyethyleneglycol may be able to satisfy the preceding conditions). Our previous studies show that for silica fiber, the biochemical layer should have a thickness in the vicinity of 1 to $15 \mathrm{~nm}$ in order to achieve highly sensitive SPR measurements. ${ }^{11}$ Therefore, for the present study, we assumed this biochemical layer to be $15 \mathrm{~nm}$ thick.

\subsection{Blood Sample Layer}

The final layer in the present SPR sensor is a very thin layer of blood sample. In general, there is no limit to the thickness of the outermost (i.e., sensing) layer for SPR-based measurements. We carried out our calculations for blood layer thickness of $1 \mathrm{~nm}$ to $1 \mu \mathrm{m}$ and there was no change in the SPR curves for three blood groups due to high optical activity of the SPW (in terms of its large penetration depth into the blood sample layer). Therefore, a very small amount of blood sample is required with the proposed scheme.

\subsection{Transmitted Power Calculations}

First, the transfer matrix method is used to calculate the reflectivity $\left(R_{p}\right)$ of the present SPR sensor design (Sec. 5). Then, the following form of angular distribution for collimated (i.e., all guided rays) launching of light in optical fiber is followed: ${ }^{11}$

$$
\mathrm{d} P \propto \frac{n_{c}^{2} \sin \theta \cos \theta}{\left(1-n_{c}^{2} \cos ^{2} \theta\right)^{2}} \mathrm{~d} \theta,
$$

where $n_{c}$ is the refractive index of the fiber core. Since any guided ray in an optical fiber may undergo multiple reflections (Fig. 1), in order to determine the effective transmitted power, the reflection coefficient $\left(R_{p}\right)$ for a single reflection is raised to the power of the number of reflections any specific propagating angle undergoes with the sensor interface. Therefore, the generalized expression for normalized transmitted power $\left(P_{\text {trans }}\right)$ in a fiber-optic SPR sensor will be:

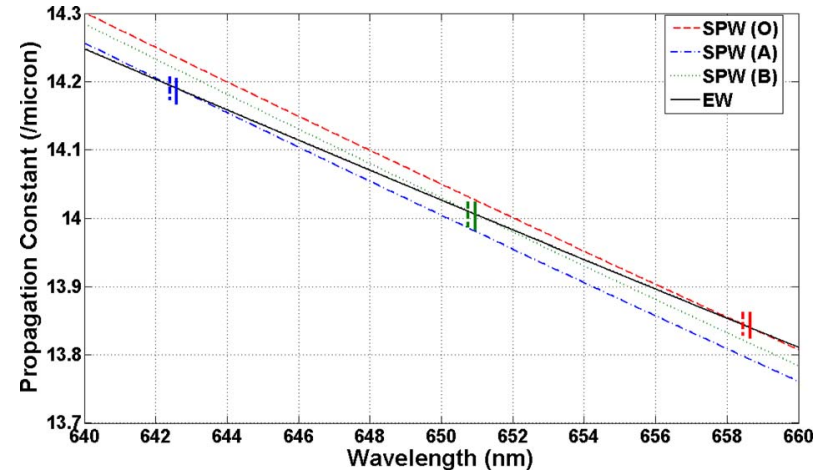

Fig. 3 Simulated results related to the fulfillment of plasmon resonance condition for three blood groups at $25^{\circ} \mathrm{C}$ and $50{ }^{\circ} \mathrm{C}$.

$$
P_{\text {trans }}=\frac{\int_{\theta_{c r}}^{\pi / 2} R_{p}^{N_{r e f}(\theta)} \frac{n_{1}^{2} \sin \theta \cos \theta}{\left(1-n_{1}^{2} \cos ^{2} \theta\right)^{2}} \mathrm{~d} \theta}{\int_{\theta_{c r}}^{\pi / 2} \frac{n_{1}^{2} \sin \theta \cos \theta}{\left(1-n_{1}^{2} \cos ^{2} \theta\right)^{2}} \mathrm{~d} \theta},
$$

where the multiple reflections $\left(N_{r e f}\right)$ of a light ray propagating inside the Ag-coated fiber core have been taken into account as follows:

$$
N_{r e f}(\theta)=\frac{L}{D \tan \theta} .
$$

$N_{\text {ref }}$ represents the total number of reflections performed by a ray making an angle $\theta$ with the normal to the fiber core-Ag layer interface. A fiber-optic probe can alternatively be used as point sensor for blood-group detection by coating one end of the fiber with an SPR-active metal layer and using the sensor in a reflection mode rather than in the present transmission mode.

\section{Results and Discussion}

In this section, we discuss the sequence of steps that were carried out to reach an optimized design of a fiber-optic SPR sensor for blood-group detection.

\subsection{Occurrence of SPR and Stability against Thermal Variation}

In order to set the optimized design parameters, the foremost task is to analyze the possibility of occurrence of SPR with the present blood-group sensing scheme. Figure 3 shows the spectral variation of $K_{S P W}$ for three blood groups $(\mathrm{O}, \mathrm{A}$, and B) along with $K_{E W}$ for fused silica fiber. The curves correspond to room temperature (i.e., $25^{\circ} \mathrm{C}$ ). As is shown in Fig. 3 , the $K_{S P W}$ curves for the three blood groups intersect the $K_{E W}$ curve at three different wavelengths $\left(\lambda_{S P R}\right)$, marked by a solid vertical line at each intersection point (i.e., $642.59 \mathrm{~nm}$ for A, $651.05 \mathrm{~nm}$ for B, and $658.57 \mathrm{~nm}$ for O blood groups). This suggests that light can be coupled to SPW to enable SPR-based detection of blood groups with the present fiber sensor scheme. The overall $\delta \lambda_{S P R}$ is $15.98 \mathrm{~nm}$, which is sufficiently large to distinguish among the three blood groups. Furthermore, we have also studied the effect of temperature 


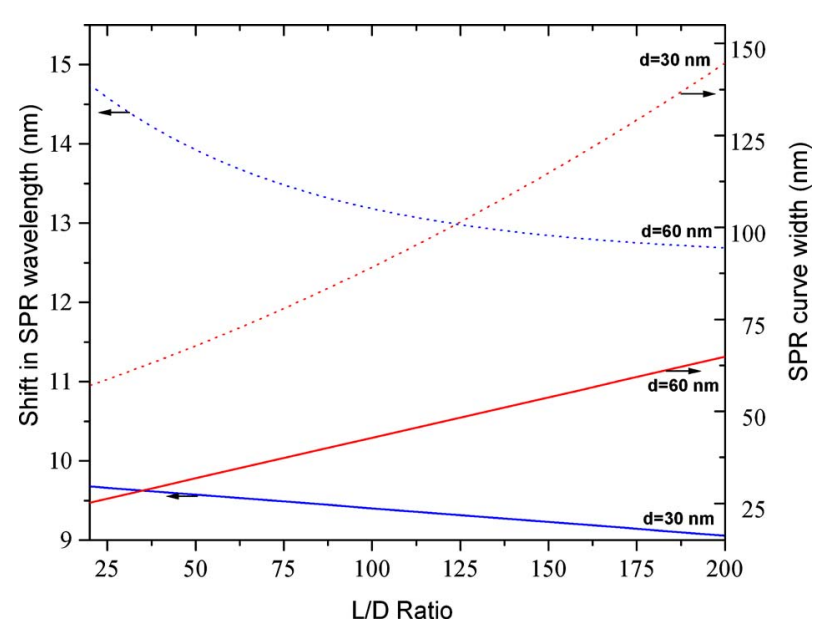

Fig. 4 Simulated master plot in order to optimize the design values of fiber core diameter $(D)$, sensing region length $(L)$, and silver layer thickness $(d)$.

variation on the preceding result by taking the thermo-optic effect in fused silica and Ag layer into account. ${ }^{12}$ The corresponding dotted vertical lines show that at $50{ }^{\circ} \mathrm{C}, \lambda_{S P R}$ values shift to the slightly shorter side (i.e., $642.36 \mathrm{~nm}$ for A, $650.85 \mathrm{~nm}$ for $\mathrm{B}$, and $658.32 \mathrm{~nm}$ for $\mathrm{O}$ blood groups). However, between $25^{\circ} \mathrm{C}$ and $50{ }^{\circ} \mathrm{C}$, the change in $\lambda_{S P R}$ is linear, small, and almost identical for all three blood groups, which suggests that there is a negligible effect of temperature variation on blood-group detection under the present fiber-optic SPR sensor scheme.

\subsection{Optimization of Design Parameters: Master Plot}

The next task is to optimize the design parameters related to optical fiber (i.e., $L$ and $D$ ) and $\mathrm{Ag}$ layer (i.e., $d$ ) in order to ensure large $\delta \lambda_{S P R}$ and small FWHM of SPR curves. From Eq. (7), it is clear that both $D$ as well as $L$ affect only the number of reflections, therefore, it is more appropriate to try to optimize the ratio $(L / D)$. Figure 4 depicts a master plot showing the effect of $L / D$ on performance parameters: overall $\delta \lambda_{S P R}$ and average FWHM. The value of $L / D$ is varied from 10 to 200. The master plot also contains the corresponding plots for two values (i.e., $30 \mathrm{~nm}$ and $60 \mathrm{~nm}$ ) of $d$. Apparently, as $L / D$ increases, the overall $\delta \lambda_{S P R}$ decreases, whereas average FWHM increases for any value of $d$. This means that with an increase in $L / D$, the sensor's performance deteriorates, which may be explained in terms of SPR-curve broadening. Each reflection a ray undergoes at the fiber-metal interface causes a certain fraction of optical power to dissipate into the sensing region due to the coupling of the evanescent wave with SPW. This further implies that larger the number of reflections, the more the decay in power $\left(P_{\text {trans }}\right)$ transmitted at the fiber's output end due to greater dissipation of power. This decay results in the downfall of SPR curve and, therefore, increases its FWHM. Since number of reflections increases with an increase in $L / D$, FWHM increases with $L / D$. Moreover, since energy-flow inside the fiber takes place in form of different discrete guided modes, which one of the modes gets coupled to SPW will depend on the precise amount of energy transferred. Further, since a mode is characterized by its angle

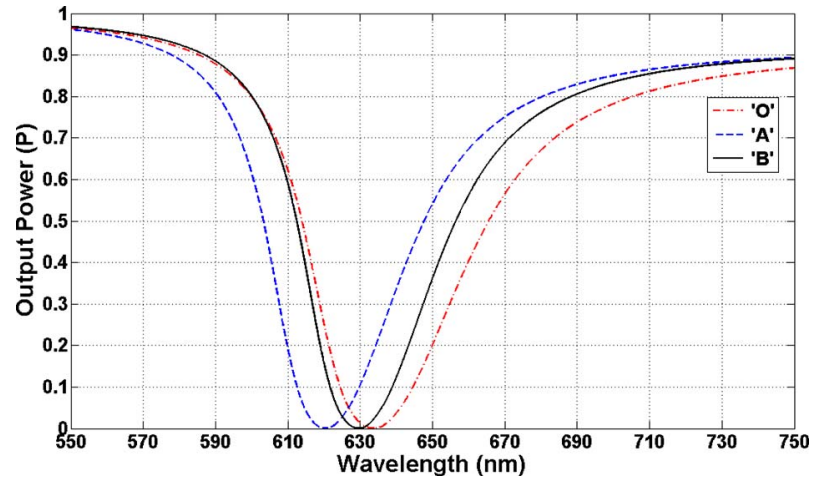

Fig. 5 Simulated SPR curves for three blood groups plotted with $L / D=10$, and $d=50 \mathrm{~nm}$ at $25^{\circ} \mathrm{C}$.

$(\theta), K_{E W}$ corresponding to the coupled mode also is affected, which in effect causes the $\lambda_{S P R}$ to shift to some other value to ensure the fulfillment of the resonance condition [see Eq. (1)]. Furthermore, since the amount of energy transfer significantly depends on $L$ and $D$ in terms of number of reflections, these two parameters ultimately affect the resonance condition also. More precisely, the resonance condition is satisfied at different wavelengths depending on what value of $L / D$ is taken.

The master plot also shows that the sensor's performance gets better with an increase in $d$. The reason is the variation in interaction between SPW and the fiber mode with a change in $d$. The thicker the Ag layer, the smaller the interaction between SPW and the fiber mode. A small interaction causes less absorption of light power, and the SPR curve shifts upward. The upshift results in narrowing of the SPR curve, and hence FWHM decreases. This variation of interaction between SPW and fiber mode also affects the resonance condition (as described earlier), and the overall $\delta \lambda_{S P R}$ increases for a thicker Ag layer.

\subsection{Demonstration of Fiber-Optic SPR Sensing for Blood-Group Detection}

Following the optimization of design parameters, Fig. 5 depicts the three SPR curves for three different blood groups $(\mathrm{O}$, $\mathrm{A}$, and B). The curves have been plotted at $25^{\circ} \mathrm{C}$ for $L / D$ $=10$ and $d=50 \mathrm{~nm}$. According to Fig. 5, the $\lambda_{S P R}$ values for the $\mathrm{A}, \mathrm{B}$, and $\mathrm{O}$ groups are $620.30 \mathrm{~nm}, 629.51 \mathrm{~nm}$, and $633.58 \mathrm{~nm}$, respectively. The preceding $\lambda_{S P R}$ values are fairly separated from one another, exhibiting an overall $\delta \lambda_{S P R}$ of $13.28 \mathrm{~nm}$ for three blood groups. The overall $\delta \lambda_{S P R}$ becomes slightly smaller than was shown in Fig. 3 because of allguided ray launching, which affects the angular distribution of the rays, and hence affects the resonance condition. Keeping in mind that a spectral shift of as small as $0.01 \mathrm{~nm}$ is commonly detectable these days, the preceding results indicate that blood-group detection with a fiber-optic SPR sensor can be conveniently carried out with high sensitivity.

Further, the sensed refractive indices of the $\mathrm{A}, \mathrm{B}$, and $\mathrm{O}$ groups at their corresponding $\lambda_{S P R}$ values (i.e., $620.30 \mathrm{~nm}$, $629.51 \mathrm{~nm}$, and $633.58 \mathrm{~nm}$, respectively) are 1.3768, 1.3788 , and 1.3796 RIU, respectively (Fig. 2). Thus, sensed refractive indices corresponding to the three blood groups are separated by $0.0028 \mathrm{RIU}$. This means that an overall $\delta \lambda_{S P R}$ of 


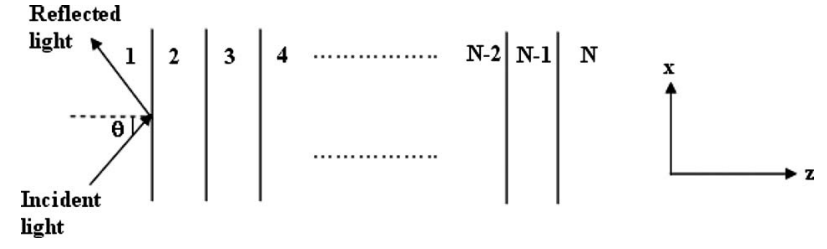

Fig. 6 Basic N-layer system to describe the transfer matrix method.

$13.28 \mathrm{~nm}$ corresponds to an overall refractive index change of 0.0028 RIU. Therefore, assuming a standard spectral resolution limit of $0.01 \mathrm{~nm}$, the theoretical detection limit with the proposed sensor design is of the order of $10^{-4}$ RIU.

Furthermore, the FWHM values for A, B, and O blood groups are $44.23 \mathrm{~nm}, \quad 38.03 \mathrm{~nm}$, and $35.04 \mathrm{~nm}$, respectively-i.e., an average FWHM of $39.10 \mathrm{~nm}$. The preceding FWHM values are in a fairly reasonable range compared to general SPR curves obtained in theoretical and experimental results.

\section{Conclusion}

A fiber-optic SPR sensor is proposed for reliable and accurate detection of human blood groups with an emphasis on achieving high sensitivity and detection accuracy. The results indicate that one should choose an optical fiber with large core diameter. Further, designing smaller SPR sensing regions with silver layer of thickness typically around $50 \mathrm{~nm}$ provides much better performance. The results indicated that the effect of temperature and stability of the SPR pattern may not be an issue on the performance of proposed sensor. Since the calculations have been carried out using experimental data, the sensor in the proposed form of a fiber-optic SPR probe as well as a point probe can be very helpful for blood-group detection in medical applications requiring only a small amount of blood. The present probe can also be reusable by using an appropriate buffer solution. Knowing the importance of blood-group detection in different medical contexts (e.g., chirurgy involving tested blood infusions), the proposed sensor, apart from differentiating the different blood groups, can open up new ways for reliable detection of overall blood properties. The proposed sensor may be cost-effective, as it requires only a broadband light source and a spectrometer for the detection.

\section{Appendix: Brief Description of Transfer Matrix Method}

A basic $N$-layer optical system is shown in Fig. 6. The layers are stacked along the $z$ axis. The arbitrary medium layer is defined by thickness $d_{k}$, dielectric constant $\varepsilon_{k}$, permeability $\mu_{k}$, and refractive index $n_{k}$. All the layers are assumed to be uniform, isotropic, and nonmagnetic. The tangential fields at the first boundary $z=z_{1}=0$ are related to those at the final boundary $z=z_{N-1}$ by:

$$
\left[\begin{array}{l}
U_{1} \\
V_{1}
\end{array}\right]=M\left[\begin{array}{l}
U_{N-1} \\
V_{N-1}
\end{array}\right]
$$

where $U_{1}$ and $V_{1}$, respectively, are the tangential components of electric and magnetic fields at the boundary of the first layer. $U_{N-1}$ and $V_{N-1}$ are the corresponding fields at the boundary of the $N^{\prime}$ th layer. Here, $M$ is known as the characteristic transfer matrix of the combined structure and is given by:

$$
M=\prod_{k=2}^{N-1} M_{k}
$$

with

$$
M_{k}=\left[\begin{array}{cc}
\cos \beta_{k} & \left(-i \sin \beta_{k}\right) / q_{k} \\
-i q_{k} \sin \beta_{k} & \cos \beta_{k}
\end{array}\right] \text {, }
$$

where

$$
q_{k}=\left(\frac{\mu_{k}}{\varepsilon_{k}}\right)^{1 / 2} \cos \theta_{k}=\frac{\left(\varepsilon_{k}-n_{1}^{2} \sin ^{2} \theta\right)^{1 / 2}}{\varepsilon_{k}}
$$

and

$$
\beta_{k}=\frac{2 \pi}{\lambda} n_{k} \cos \theta_{k}\left(z_{k}-z_{k-1}\right)=\frac{2 \pi d_{k}}{\lambda}\left(\varepsilon_{k}-n_{1}^{2} \sin ^{2} \theta\right)^{1 / 2}
$$

The amplitude reflection coefficient $\left(r_{p}\right)$ is given by

$$
r_{p}=\frac{\left(M_{11}+M_{12} q_{N}\right) q_{1}-\left(M_{21}+M_{22} q_{N}\right)}{\left(M_{11}+M_{12} q_{N}\right) q_{1}+\left(M_{21}+M_{22} q_{N}\right)}
$$

Last, the reflectivity $\left(R_{p}\right)$ is calculated as

$$
R_{p}=\left|r_{p}\right|^{2}
$$

In the present sensor design, we have a total of four layers (fiber core, Ag layer, buffer layer, and sample blood layer), i.e., $N=4$. So, by using of the values of refractive index and the thickness of the different layers as discussed earlier, the value of reflectivity $\left(R_{p}\right)$ can be conveniently calculated with the help of the transfer matrix method for further use in obtaining the transmitted power $\left(P_{\text {trans }}\right)$, as discussed in Sec. 2.5 .

\section{Acknowledgments}

Anuj Kumar Sharma would like to thank the Alexander von Humboldt Foundation (Germany) for financial support during his research stay at Friedrich-Schiller University Jena (Germany). Gerhard J. Mohr was supported by Deutsche Forschungsgemeinschaft research grants MO 1062/5-1 and MO 1062/6-1.

\section{References}

1. I. Pockrand, J. D. Swalen, J. G. Gordan, and M. R. Philpott, "Surface plasmon spectroscopy of organic monolayer assemblies," Surf. Sci. 74, 237-244 (1978).

2. B. Liedberg, C. Nylander, and I. Sundstrom, "Surface plasmon resonance for gas detection and biosensing," Sens. Actuators 4, 299-304 (1983).

3. R. C. Jorgenson and S. S. Yee, "A fiber-optic chemical sensor based on surface plasmon resonance," Sens. Actuators B 12, 213-220 (1993).

4. T. Okamoto, I. Yamaguchi, and T. Kobayashi, "Local plasmon sensor with gold colloid monolayers deposited upon glass substrates," Opt. Lett. 25, 372-374 (2000). 
5. R. Jha, S. Chand, and B. D. Gupta, "Surface plasmon resonance based fiber-optic sensor for detection of pesticide," Sens. Actuators $B$ 123, 661-666 (2007).

6. E. Kretchmann and H. Reather, "Radiative decay of non-radiative surface plasmons excited by light," Z. Naturforsch [C] 23, 21352136 (1968).

7. Z. Salamon, H. A. Macleod, and G. Tollin, "Surface plasmon resonance spectroscopy as a tool for investigating the biochemical and biophysical properties of membrane protein systems. II: Applications to biological systems," Biochim. Biophys. Acta, 1331, 131-152 (1997).

8. E. Matveeva, J. Malicka, I. Gryczynski, Z. Gryczynski, and J. R. Lakowicz, "Multi-wavelength immunoassays using surface plasmoncoupled emission," Biochem. Biophys. Res. Commun. 313, 721-726
(2004).

9. H. Li, L. Lin, and S. Xie, "Refractive index of human whole blood with different types in the visible and near-infrared ranges," Proc. SPIE, 3914, 517-521 (2000).

10. A. Ghatak and K. Thyagarajan, "Material dispersion," Chapter 6 in An Introduction to Fiber Optics, pp. 80-82, Cambridge University Press, Cambridge, UK (1998).

11. B. D. Gupta and A. K. Sharma, "Sensitivity evulation of a multilayered surface plasmon resonance based fiber optic sensor: a theoretical study," Sens. Actuators B 107, 40-46 (2005).

12. A. K. Sharma and B. D. Gupta, "Influence of temperature on the sensitivity and signal-to-noise ratio of fiber optic surface plasmon resonance sensor," Appl. Opt. 45, 151-161 (2006). 